\title{
A quantitative analysis of craniopharyngioma cyst expansion during and after radiation therapy and surgical implications
}

\author{
Kelly Lamiman, BS, ${ }^{1}$ Kenneth K. Wong, MD, ${ }^{2}$ Benita Tamrazi, MD, ${ }^{3}$ Jason D. Nosrati, BA, ${ }^{5}$ \\ Arthur Olch, PhD, ${ }^{2}$ Eric L. Chang, MD, ${ }^{6}$ and Erin N. Kiehna, MD ${ }^{4}$ \\ 1 University of Cincinnati College of Medicine, Cincinnati, Ohio; ${ }^{2}$ Department of Radiation Oncology, ${ }^{3}$ Department of Radiology, \\ Departments of ${ }^{4}$ Neurosurgery and ${ }^{6}$ Radiation Oncology, Children's Hospital Los Angeles, Keck School of Medicine of USC, \\ University of Southern California, Los Angeles, California; ${ }^{5}$ Michigan State University College of Human Medicine, Grand Rapids, \\ Michigan
}

OBJECTIVE When complete resection of craniopharyngioma is not achievable or the sequelae are prohibitive, limited surgery and radiation therapy have demonstrated excellent local disease control while minimizing treatment-related sequelae. When residual tissue exists, there is a propensity for further cyst development and expansion during and after radiation therapy. This can result in obstructive hydrocephalus, visual changes, and/or clinical decline. The authors present a quantitative analysis of cyst expansion during and after radiotherapy and examine how it affected subsequent management.

METHODS The authors performed an institutional review board-approved retrospective study of patients with histologically confirmed craniopharyngioma treated between 2000 and 2015 with surgery and intensity-modulated radiation therapy (IMRT) at a single institution. Volumetric measurements of cyst contours were generated by radiation oncology treatment planning software postoperatively, during IMRT, and up to 12 months after IMRT. Patient, tumor, and treatment-related variables were collected until the last known follow-up and were analyzed.

RESULTS Twenty-seven patients underwent surgery and IMRT. The median total radiation dose was 54 Gy. Of the 27 patients, 11 patients (40.7\%) demonstrated cyst expansions within 1 year of IMRT. Of note, all tumors with cyst expansion were radiographically Puget Grade 2. Maximal cyst expansion peaked at 4.27 months following radiation therapy, with a median volume growth of $4.1 \mathrm{~cm}^{3}$ (mean $9.61 \mathrm{~cm}^{3}$ ) above the postoperative cyst volume. Eight patients experienced spontaneous cyst regression without therapeutic intervention. Three patients experienced MRI-confirmed cyst enlargement during IMRT, all of whom required adaptive planning to ensure adequate coverage of the entire tumor volume. Two of these 3 patients required ventriculoperitoneal shunt placement and additional intervention. One underwent additional resection, and the other had placement of an intracystic catheter for aspiration and delivery of intracystic interferon within 12 months of completing IMRT. All 3 patients now have stable disease.

CONCLUSIONS Craniopharyngioma cyst expansion occurred in approximately $40 \%$ of the patients during or after radiotherapy. In the majority of patients, cyst expansion was a self-limiting process and did not confer a worse outcome. During radiotherapy, cyst expansion may be apparent on image-guided radiation therapy. Adaptive IMRT planning may be required to ensure that the intended IMRT dose covers the entire tumor and cyst volume. The sequelae of cyst expansion include progressive hydrocephalus, which may be treated with a shunt. For patients with solitary cyst expansion, cyst aspiration and/or intracystic interferon may result in disease control.

https://thejns.org/doi/abs/10.3171/2016.9.FOCUS16298

KEY WORDS craniopharyngioma; radiotherapy; intensity-modulated radiation therapy; image-guided radiation therapy; hydrocephalus; cysts 


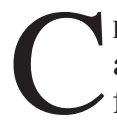
RANIOPHARYNGIOMA is a benign epithelial tumor that arises in the sellar or suprasellar region of the brain from embryological remnants of Rathke's pouch. ${ }^{6}$ The peak age at diagnosis for pediatric craniopharyngioma is $8-10$ years old. ${ }^{2}$ Due to the tumor's propensity to arise close to the optic chiasm, pituitary gland, hypothalamus, and third ventricle, a craniopharyngioma can cause significant mass effect symptoms. Furthermore, if the tumor invades adjacent structures, resection could result in significant morbidity.

Primary treatment paradigms for craniopharyngioma include resection with or without adjuvant radiotherapy. When lesions are predominantly cystic, complementary treatment options include Ommaya reservoir placement, cyst drainage, and intracystic injectable agents.,17 Treatment paradigms depend on tumor composition, location, and extent of invasion into adjacent structures. In 2007, Puget et al. reviewed their treatment experience at Hôpital Necker-Enfants Malades, Paris, France, and designed an MRI grading scale for craniopharyngioma based on the degree of hypothalamic involvement. ${ }^{13}$ They defined Grade 0 lesions as small and/or subdiaphragmatic tumors without hypothalamic involvement, Grade 1 lesions as tumors that abut or displace the hypothalamus, and Grade 2 lesions as tumors with hypothalamic involvement and/or unidentifiable hypothalamus (Table 1). For Puget Grade 0 tumors, gross-total resection (GTR) of the tumor can often be achieved; when GTR is achieved, radiation therapy may be delayed or avoided. For Puget Grade 1 lesions displacing the hypothalamus or Puget Grade 2 lesions involving the hypothalamus, there is a higher risk for disturbing normal structures during an attempt at complete resection. In these cases, the surgical goal may be a subtotal resection (STR) or near-total resection (NTR) to relieve local compressive symptoms while reducing the risk of operative morbidity and mortality. ${ }^{7}$ When there is incomplete resection, radiotherapy is often added as adjuvant therapy to reduce the risk of recurrence. Incompletely resected craniopharyngiomas are likely to progress within 1 year without adjuvant radiotherapy. ${ }^{15}$

There is no clear consensus regarding the best primary therapy to treat craniopharyngioma. With partial resection and radiation therapy, the 10-year local control rate is comparable to GTR only (77\%-100\%). ${ }^{8}$ At our institution, the current approach is to attempt the maximal safe resection to avoid or postpone the need for radiation therapy and reduce exposure to the potential for radiation-induced sequelae, including secondary malignancies, endocrinopathies, and neurocognitive impairment. When GTR is not possible, our approach shifts to partial resection with planned adjuvant intensity-modulated radiotherapy (IMRT). When a patient's tumor recurs following radiotherapy, options are discussed at a multidisciplinary tumor board.

Several studies have reported cyst enlargement of the tumor during radiation therapy and in the months immediately following radiation therapy in as many as 30\%$60 \%$ of craniopharyngioma patients..$^{2,5,9,14,16}$ Previously, it was thought that any cyst enlargement was a sign of recurrence and thus required surgical intervention. However, cyst enlargement seen during or after radiotherapy is often followed by regression without the need for ad-
TABLE 1. Craniopharyngioma radiographic grading scale

\begin{tabular}{cl}
\hline Puget Grade & \multicolumn{1}{c}{ Definition } \\
\hline 0 & $\begin{array}{c}\text { No hypothalamic involvement or displacement on } \\
\text { diagnostic MRI }\end{array}$ \\
\hline 1 & Hypothalamus displaced by tumor on diagnostic MRI \\
\hline 2 & Hypothalamic involvement on diagnostic MRI \\
\hline
\end{tabular}

As defined by Puget et al.

ditional therapeutic intervention. ${ }^{5}$ When cyst enlargement occurs during radiation therapy, it may warrant adaptive treatment plan modifications to ensure adequate IMRT coverage. Thus, accurate methods of predicting, detecting, and tracking cyst enlargement during and after IMRT are needed to ensure adequate coverage, reduce the risk of recurrence, and guide decisions regarding the need for additional surgical intervention.

\section{Methods \\ Study Population}

This study was conducted with the approval of the institutional review board at Children's Hospital Los Angeles (CHLA). Patients were identified on the basis of a keyword search. Medical records of pediatric and young adult patients undergoing neurosurgical resection at CHLA for histopathologically confirmed craniopharyngioma between 2000 and 2015 were retrospectively reviewed, and a total of 80 patients were identified (Fig. 1). Forty-nine patients did not receive radiation therapy or received radiation therapy outside our facility and thus were excluded from our analysis. An additional 4 patients underwent primary resection elsewhere and were excluded due to incomplete records. A total of 27 patients who underwent primary surgery and IMRT at our institution were included in our analysis.

\section{Classification and Grading}

Each patient underwent neurosurgical resection and had a histologically confirmed craniopharyngioma. The extent of resection was determined on the basis of the operative reports and postoperative MRI results. GTR was rigidly defined as complete tumor removal, NTR was defined as 90\%-99.9\% tumor removal, and STR was defined as 50\%-90\% removal. Treatment decisions, including referrals to radiation therapy, were determined on a case-by-case basis by a multidisciplinary tumor board. Craniopharyngioma lesions were graded based on MRI according to the classification system outlined by Puget et al. (Table 1). ${ }^{13}$

\section{Imaging and Follow-Up}

All 27 patients underwent initial surgery at CHLA and were monitored during and after IMRT at CHLA. Follow-up brain MR images with and without contrast were obtained within 72 hours of surgery, at approximately 1 month post-IMRT, every 3-4 months until 1 year postIMRT, every 4-6 months until 2 years post-IMRT, and annually thereafter. Cases with cyst enlargement alone 


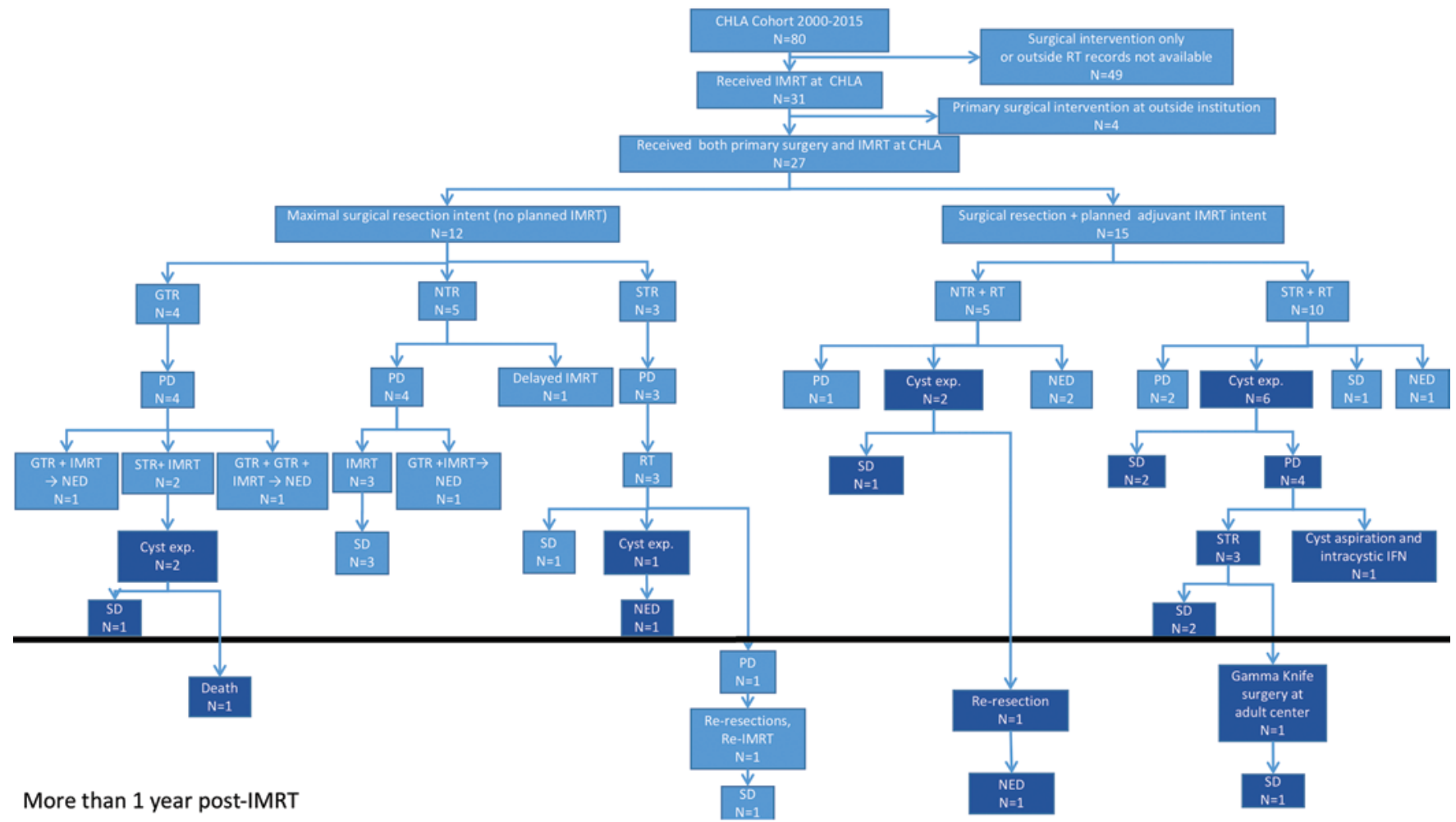

FIG. 1. Treatment paradigms of patients receiving treatment at CHLA for craniopharyngioma through the last follow-up. Cyst exp. = cyst expansion; IFN = interferon; NED = no evidence of disease; $\mathrm{PD}=$ progressive disease; $\mathrm{RT}=$ radiation therapy; $\mathrm{SD}=$ stable disease.

(in the absence of solid tumor progression) on MRI during or after IMRT that spontaneously regressed without intervention were not categorized as progressive disease. When cyst enlargement is suspected during IMRT because of symptoms, or cone beam CT (CBCT) imaging guidance, our institutional policy is to perform a confirmatory MRI.

All MR images were imported into radiation oncology treatment planning software (Eclipse, Varian Medical Systems) to volumetrically contour the craniopharyngioma cysts postoperatively, during IMRT, and up to 12 months following IMRT. If additional surgical intervention occurred within 12 months following IMRT, those patients became censored. Two patients received ventriculoperitoneal (VP) shunts during IMRT and were included in the analysis until the time of additional surgical intervention. Volumetric measurements were automatically generated by the Eclipse software after manual contouring of the tumor cyst as seen on T2-weighted MRI sequences.

\section{Radiation Treatment Planning}

Patients received fractionated non-coplanar IMRT using a HeadFIX (Elekta AB) custom head immobilization and frameless mouthpiece. Treatment planning during 2000-2006 was performed using PLATO (Nucletron Corp.), and treatment planning during 2006-2015 was performed using the Eclipse system. Image guidance with CBCT has been used during IMRT treatment since 2013.

\section{Statistical Analysis}

All statistical analyses were performed using Microsoft Excel and Prism for Windows (version 7.0a, GraphPad Software, Inc.); $\mathrm{p}$ values for Kaplan-Meier curves were calculated using the log-rank (Mantel-Cox) test. Overall survival (OS) and progression-free survival (PFS) were calculated from the end of IMRT.

\section{Results}

\section{Patient and Treatment Characteristics}

Table 2 summarizes the demographics of patients included in this study and their treatment protocols. Of the 27 patients, 16 were male $(59.3 \%)$ and 11 were female $(40.7 \%)$. The median age at diagnosis was 9.9 years (range 2.7-17.6 years). Fifteen patients (55.6\%) had adjuvant IMRT immediately after STR or NTR, and 12 patients (44.4\%) received salvage IMRT at recurrence. The median radiation dose was 54 Gy (range 52-59.4 Gy) given in a median of 30 fractions (range 29-33 fractions). All patients had IMRT plans with a median of 8 non-coplanar beams (range 5-9 beams). The median gross target volume (GTV) was $18.2 \mathrm{~cm}^{3}$ (range $1.5-240.9 \mathrm{~cm}^{3}$ ). Clinical target volume margins ranged from 5 to $10 \mathrm{~mm}$ outside of the GTV, and the median planned target volume (PTV) margins were $3 \mathrm{~mm}$ outside of the clinical target volume. The median follow-up time after the end of IMRT was 58 months (range 6-123 months). Twenty-six of 27 patients (96.3\%) were alive at last follow-up. 
TABLE 2. Patient, tumor, and treatment-related variables in 27 patients who underwent surgery and IMRT

\begin{tabular}{lc}
\hline \multicolumn{1}{c}{ Variable } & Value \\
\hline Sex & \\
M & $16(59.3 \%)$ \\
F & $11(40.7 \%)$ \\
\hline Race & \\
Hispanic & $21(77.8 \%)$ \\
Non-Hispanic white & $3(11.1 \%)$ \\
Asian & $2(7.4 \%)$ \\
Non-Hispanic black & $1(3.7 \%)$ \\
\hline Median age at diagnosis in yrs (range) & $9.9(2.7-17.6)$ \\
\hline Median time from 1st op to IMRT in mos (range) & $1.3(0.7-198.2)$ \\
\hline Primary operative outcome & $4(14.8 \%)$ \\
GTR & $9(33.3 \%)$ \\
NTR & $14(50 \%)$ \\
STR & \\
\hline Puget Grade & $0(0 \%)$ \\
0 & $6(22.2 \%)$ \\
1 & $21(77.8 \%)$ \\
2 & \\
\hline IMRT & $15(55.6 \%)$ \\
Planned adjuvant & $12(44.4 \%)$ \\
At recurrence & $5400(5200-5940)$ \\
\hline Median IMRT dose (for 1st course), cGy (range) & $30(29-33)$ \\
\hline Median no of fractions (for 1st course) (range) & $18.2(1.5-240.9)$ \\
\hline Median GTV (on initial plan) in cm ${ }^{3}$ (range) & 8 \\
\hline Median no. of non-coplanar beams & \\
\hline
\end{tabular}

\section{Cyst Volume Changes During and After IMRT}

Eleven of 27 patients $(40.7 \%)$ demonstrated cyst expansion noted on MRI and confirmed with volumetric contours during or within 12 months of completing IMRT (Fig. 2 and Table 3). Of note, all tumors with cyst enlargement were classified as a Puget Grade 2 craniopharyngioma. The median postoperative, pre-IMRT cyst volume was not prognostic in determining which patients would go on to experience cyst enlargement $\left(0.75 \mathrm{~cm}^{3}\right.$ for those without cyst growth vs $1.9 \mathrm{~cm}^{3}$ for those with cyst growth, $\mathrm{p}=0.628$ ). Maximum cyst expansion peaked at a median of 4.27 months following IMRT (mean 4.94 months) with a median volume increase of $4.1 \mathrm{~cm}^{3}$ (mean $9.61 \mathrm{~cm}^{3}$ ) (Fig. 3 ) above the postoperative cyst volume. Seven patients experienced spontaneous cyst regression without therapeutic intervention, with a median volume of only $0.1 \mathrm{~cm}^{3}$ above the postoperative cyst volume without intervention by 12 months following IMRT. For the 7 patients with self-limited cyst expansion, only 2 developed an additional endocrinopathy in the year following radiation therapy (permanent hypothyroidism and growth hormone deficiency). Both could have been caused by radiation therapy alone versus cyst expansion. Figure 4 demonstrates self-limited cyst expansion in a patient who maintains normal visual acuity, normal visual fields, and an intact hypothalamic pituitary axis (Patient 1).

Three of 11 patients with cyst expansion required surgical intervention. Patient 2 returned to his home country less than 1 year following IMRT and could not receive further care. We were notified of his death 2 years after he completed radiation therapy. Patient 5 experienced cyst progression (increase of $16 \mathrm{~cm}^{3}$ ) following IMRT, requiring a return to the operating room for STR at 134 days post-IMRT. He had panhypopituitarism at presentation. Patient 9 developed symptoms of hydrocephalus 4 days after the start of radiation therapy, and MRI revealed interval cyst growth of $8 \mathrm{~cm}^{3}$. Her hydrocephalus was treated with a VP shunt. The cyst continued to expand following IMRT with visual deterioration, and the patient underwent placement of an Ommaya reservoir for cyst aspiration and received intracystic interferon at 152 days post-IMRT. There was a complete response with a 15-month follow-up. There was no change in hypothalamic pituitary function. Patient 10 developed symptoms of hydrocephalus near the end of radiotherapy, and MRI demonstrated cyst growth of $27 \mathrm{~cm}^{3}$ requiring VP shunt placement 5 days before the conclusion of IMRT. He experienced further cyst progression within the temporal lobe and underwent STR at 142 days post-IMRT. During this interval the patient was diagnosed with hypogonadism, in addition to preexisting hormone deficiencies. The patients have not required subsequent surgeries since these additional surgical interventions.

Three patients had MRI-confirmed cyst enlargement during IMRT that required adaptive planning to ensure adequate coverage of the GTV. Two of those patients went on to receive additional surgical intervention as previously described (Patients 9 and 10), and one experienced spontaneous regression (Patient 1).

\section{Treatment Outcomes}

Cyst enlargement during or after IMRT was not prognostic in determining OS $(100 \%$ for those without cyst enlargement vs $90.9 \%$ for those with cyst enlargement, $\mathrm{p}$ $=0.14$; Fig. $5 \mathrm{~A})$, or PFS (54.5\% for those without cyst enlargement vs $56.3 \%$ for those with cyst enlargement, $\mathrm{p}=$ 0.82 ; Fig. 5B).

The Puget lesion grade at diagnosis was the only statistically significant factor for PFS $(100 \%$ for Puget Grade 1 lesions vs $45.1 \%$ for Puget Grade 2 lesions, $p=0.05$; Fig. $5 \mathrm{C}$ and Table 1). There is not a statistically significant difference in PFS by treatment intent $(81.5 \%$ for those with maximal surgery and delayed IMRT vs $39.9 \%$ for combined surgery and IMRT, $\mathrm{p}=0.07$; Fig. 5D), which may be accounted for by a greater percentage of patients with Puget Grade 1 lesions treated in the surgery-alone cohort $(33 \%, 4$ of 12$)$ versus limited surgery with planned IMRT $(13 \%, 2$ of 15$)$.

\section{Discussion}

Solid and cystic tumor progression is known to occur during radiation therapy, affecting nearly half of all patients. ${ }^{11}$ Understanding the mechanisms behind radiationinduced tumor death may explain why cysts enlarge during or immediately following treatment. Radiotherapy induces tumor cell death via free radical formation, leading cells to lose the ability to function or sustain proliferation. However, irradiated tumor cells may remain intact 


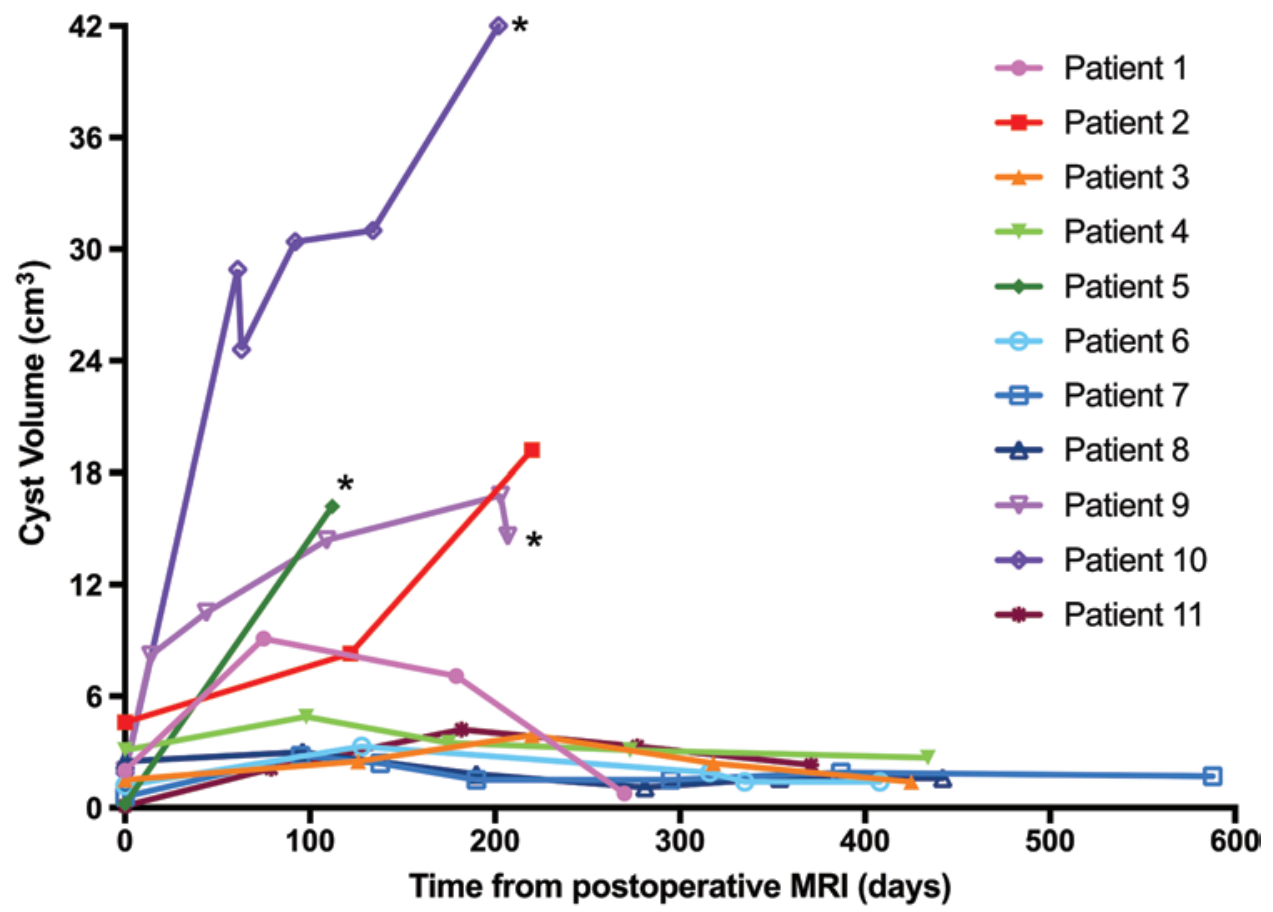

FIG. 2. Graph showing the changes in cyst volume from the postoperative MRI session to 12 months after radiation therapy. Patients requiring additional surgical intervention were censored at that time. Asterisks indicate patients who required surgical intervention within 12 months following IMRT.

and may be capable of a few additional replication cycles before their eventual demise..$^{11}$ Radiation can also cause late changes in the osmotic gradient and permeability of the cyst wall, which may contribute to production of additional fluid by residual tumor or CSF crossing into the cyst. ${ }^{6}$ Inflammation mediated by $\alpha$-defensins has also been implicated in cyst formation. ${ }^{2}$ Given that the majority of craniopharyngiomas are slow growing, it is plausible that radiation-induced cell death may lead to a temporary increase in tumor size over the several months during and after radiotherapy. This theory is supported by the evolution of cyst volumes measured before, during, and after radiotherapy in our patients. Additional study of the histopathological features of cyst lesions is warranted to determine if correlations exist between the type of cell lining or cyst contents and the incidence of cyst enlargement during or after radiotherapy.

Cyst enlargement in response to radiation poses $2 \mathrm{ma}-$

TABLE 3. Patient, tumor, and treatment characteristics of patients with cystic expansion during and after IMRT

\begin{tabular}{|c|c|c|c|c|c|c|c|c|c|c|}
\hline $\begin{array}{l}\text { Patient } \\
\text { No. }\end{array}$ & $\begin{array}{c}\text { Age (yrs) at } \\
\text { Diagnosis, } \\
\text { Sex }\end{array}$ & $\begin{array}{l}\text { Puget } \\
\text { Grade }\end{array}$ & $\begin{array}{c}\text { Extent } \\
\text { of 1st } \\
\text { Resection }\end{array}$ & $\begin{array}{c}\text { Planned } \\
\text { Adjuvant } \\
\text { RT }\end{array}$ & $\begin{array}{c}\text { Mos From } \\
\text { 1st Op to } \\
\text { IMRT }\end{array}$ & $\begin{array}{l}\text { GTV } \\
\left(\mathrm{cm}^{3}\right)\end{array}$ & $\begin{array}{l}\text { Additional Cyst } \\
\text { Expansion }\left(\mathrm{cm}^{3}\right)\end{array}$ & $\begin{array}{c}\text { Intervention } \\
\text { During RT }\end{array}$ & $\begin{array}{l}\text { Intervention } \\
\text { w/in } 12 \text { Mos } \\
\text { Post-IMRT }\end{array}$ & $\begin{array}{l}\text { Disease } \\
\text { Status at } \\
\text { Last FU }\end{array}$ \\
\hline 1 & $4.9, \mathrm{M}$ & 2 & STR & Yes & 0.97 & 9.5 & 7.1 & None & None & SD \\
\hline 2 & 3.9, M & 2 & GTR & No & 80.17 & 39.8 & 14.6 & None & None & Death* \\
\hline 3 & $8.8, F$ & 2 & STR & Yes & 1.03 & 23.4 & 2.4 & None & None & SD \\
\hline 4 & 7.3, M & 2 & STR & No & 17.00 & 6.5 & 1.8 & None & None & NED \\
\hline 5 & $16.3, \mathrm{M}$ & 2 & STR & Yes & 1.33 & 53.7 & 16 & None & STR & SD \\
\hline 6 & $7.4, \mathrm{M}$ & 2 & STR & Yes & 1.23 & 13.5 & 2.1 & None & None & SD \\
\hline 7 & 2.7, M & 2 & GTR & No & 198.23 & 2.8 & 2.2 & None & None & SD \\
\hline 8 & $9.7, \mathrm{~F}$ & 2 & STR & Yes & 0.90 & 15.7 & 0.5 & None & None & SD \\
\hline 9 & $12.0, \mathrm{~F}$ & 2 & STR & Yes & 0.70 & 18.2 & 14.8 & VP shunt & $\begin{array}{l}\text { Cyst aspiration \& in- } \\
\text { tracystic interferon }\end{array}$ & SD \\
\hline 10 & $14.9, \mathrm{M}$ & 2 & STR & Yes & 0.93 & 24 & 40.1 & VP shunt & STR & SD \\
\hline 11 & $6.9, \mathrm{M}$ & 2 & NTR & Yes & 1.30 & 13.8 & 4.1 & None & None & NED \\
\hline
\end{tabular}

$\mathrm{FU}=$ follow-up; NED = no evidence of disease; RT = radiation therapy; $\mathrm{SD}=$ stable disease.

* Patient returned to home country and was not allowed to return to US. We were subsequently notified of his death. 


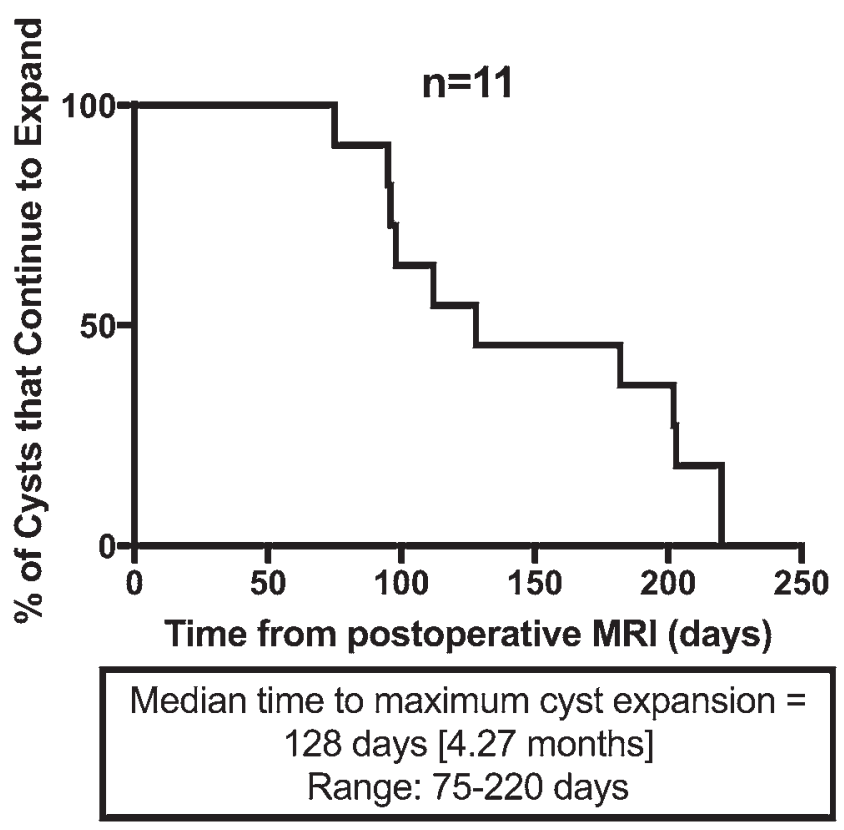

FIG. 3. Kaplan-Meier curve of time to maximal cyst expansion in the patients experiencing enlargement during and after IMRT $(n=11)$. Each event denotes the date of maximal expansion determined by volumetric contour of MRI.

jor concerns for the radiation oncology and neurosurgical teams. When cysts enlarge during radiation therapy, there is concern for inadequate coverage and underdosing portions of the tumor. Additionally, there is question about the need for additional neurosurgical intervention as the cyst enlarges. Bishop et al. reported a $40 \%$ incidence in cyst growth on treatment requiring intervention in $20 \% .{ }^{4}$ Previous reports indicated that there was no correlation between initial preradiotherapy cyst volume and the probability of cyst expansion. ${ }^{11}$ We examined the Puget grade for each tumor and demonstrated that Puget Grade 2 lesions have a greater risk for cyst enlargement during and within the few months following IMRT. Additionally, patients with Puget Grade 2 lesions may have a higher risk of requiring surgical intervention post-IMRT. In these cases, it is important to closely follow tumor changes and confirm suspected changes with diagnostic imaging. If cyst enlargement occurs within the few months immediately following IMRT, the role for additional neurosurgical intervention remains difficult to determine in otherwise asymptomatic patients. There are previous reports of visual and hypothalamic toxicity related to cyst growth; ${ }^{4}$ however, this was not witnessed in our patient population.

The rate of cyst enlargement in patients treated at our institution $(40.7 \%, 11$ of 27 patients) falls within the range reported in existing literature of approximately 30\%$62 \%{ }^{3-5,10,14,16}$ Cyst enlargement caused recurrent hydrocephalus in 2 patients whose ventriculomegaly originally resolved following surgery, requiring treatment with a VP shunt. However, we demonstrate that the majority of cyst changes are self-limiting, in concordance with the literature. ${ }^{4}$ Our study suggests that cyst expansion occurs exclusively in Puget Grade 2 craniopharyngioma. Therefore, it is reasonable to recommend additional surveillance for cyst enlargement in this tumor population. Two patients early in our series underwent additional STR for treatment of progressive cyst enlargement following IMRT. The most recent patient-and future patients with solitary cyst expansion-is being treated with placement of a cyst catheter, aspiration, and intracystic interferon with prom-

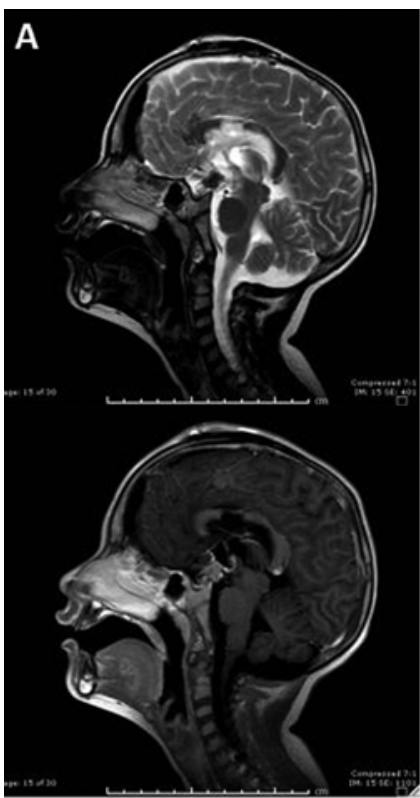

Postoperative

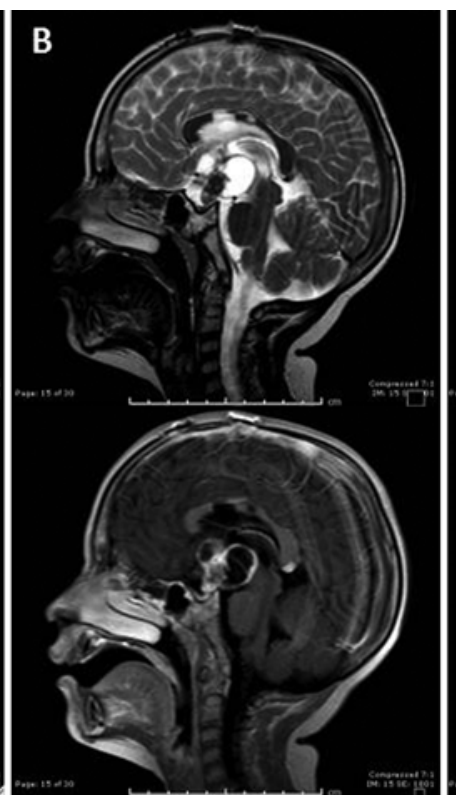

3 months Post-IMRT

$7 \mathrm{~cm}^{3}$ cyst volume

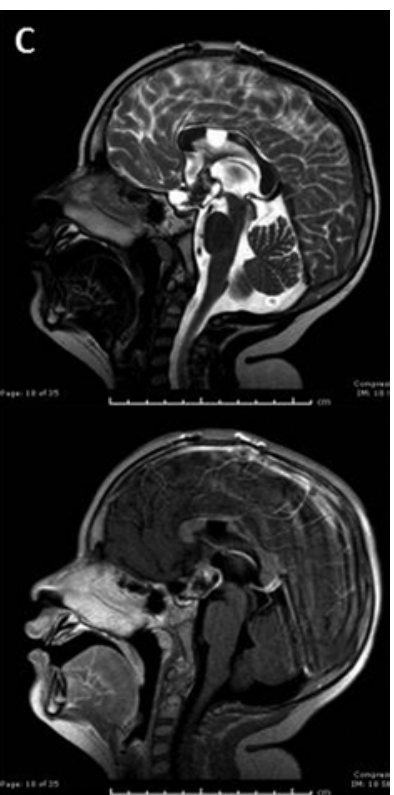

6 months Post-IMRT $1 \mathrm{~cm}^{3}$ cyst volume

FIG. 4. Representative example of self-limited cyst enlargement visualized from postoperative sagittal MR images (A) to 3 months post-IMRT (B) to near resolution 6 months post-IMRT (C). 
Overall Survival

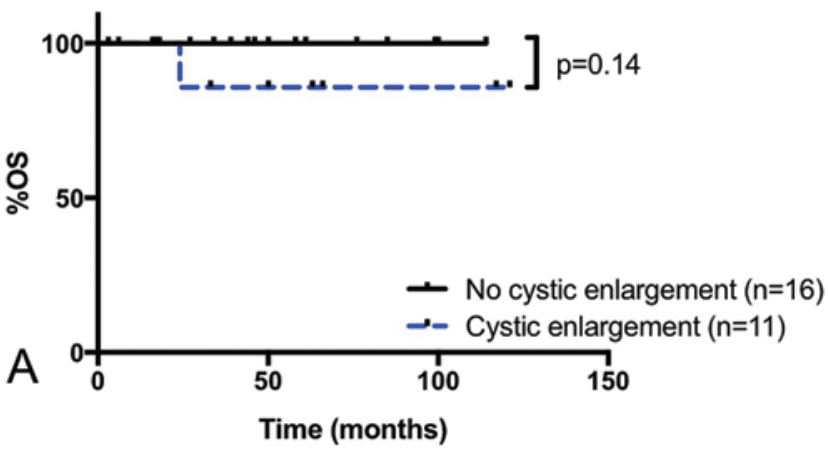

PFS:

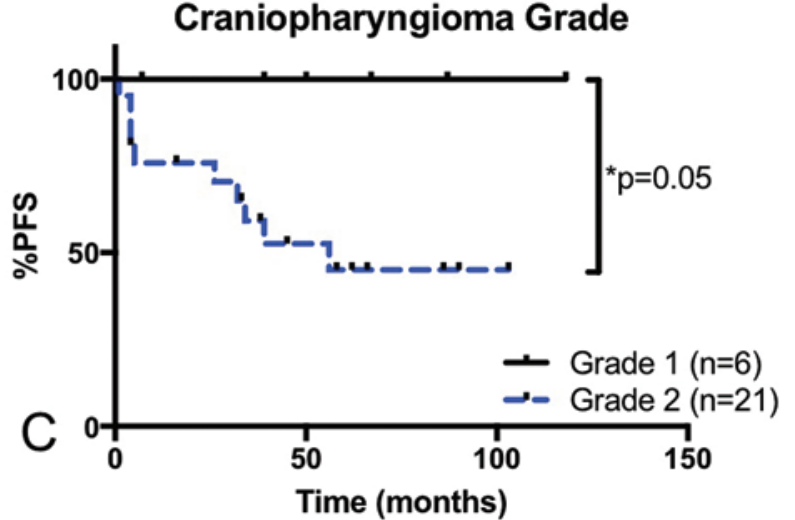

PFS:

Cystic Enlargement

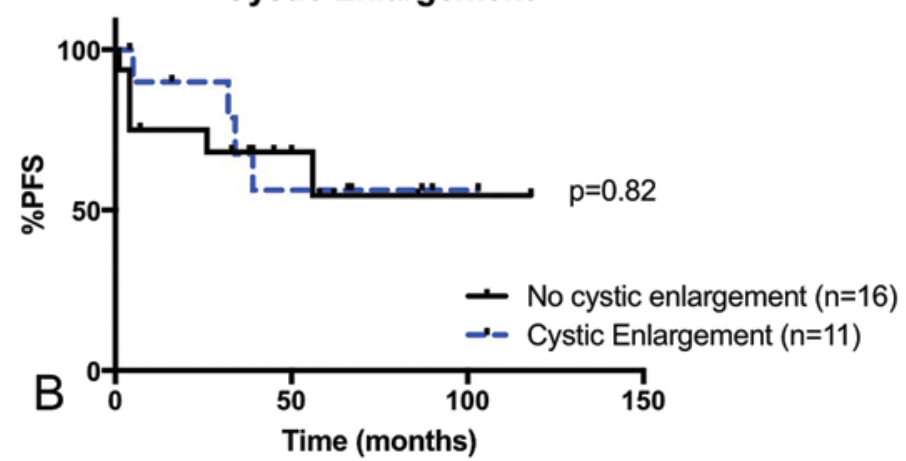

PFS:

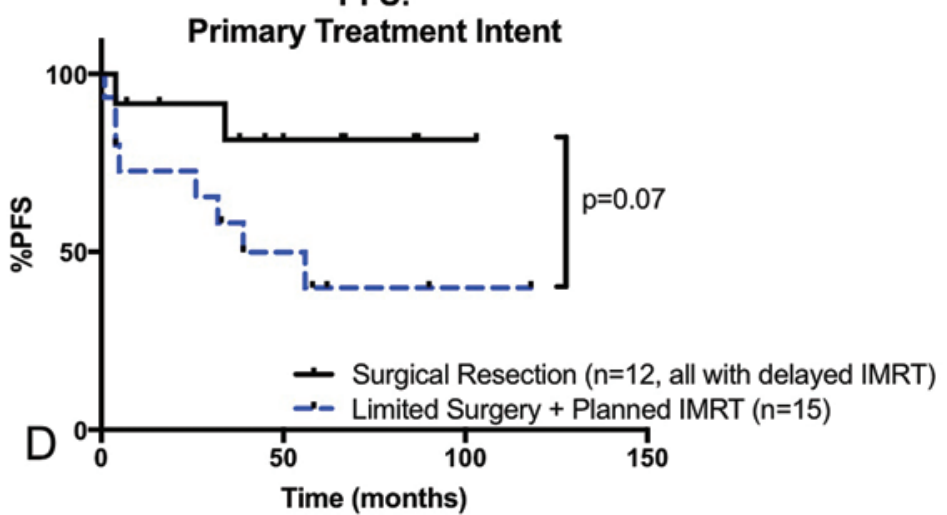

FIG. 5. Survival analysis following IMRT. OS (A) and PFS (B) based on cyst enlargement. PFS based on the Puget et al. grading scale (C). PFS based on primary treatment intent (D).

ising results (Fig. 6). This is a minimally invasive way to stabilize the cyst volume and may result in a therapeutic advantage over radiation alone.

Cyst enlargement can be potentially identified dur- ing IMRT using daily image-guided radiation therapy to detect changes in size and location of residual calcified tumor components. In a small subset of 3 patients, Kornguth et al. showed that weekly CT-on-rails images fused
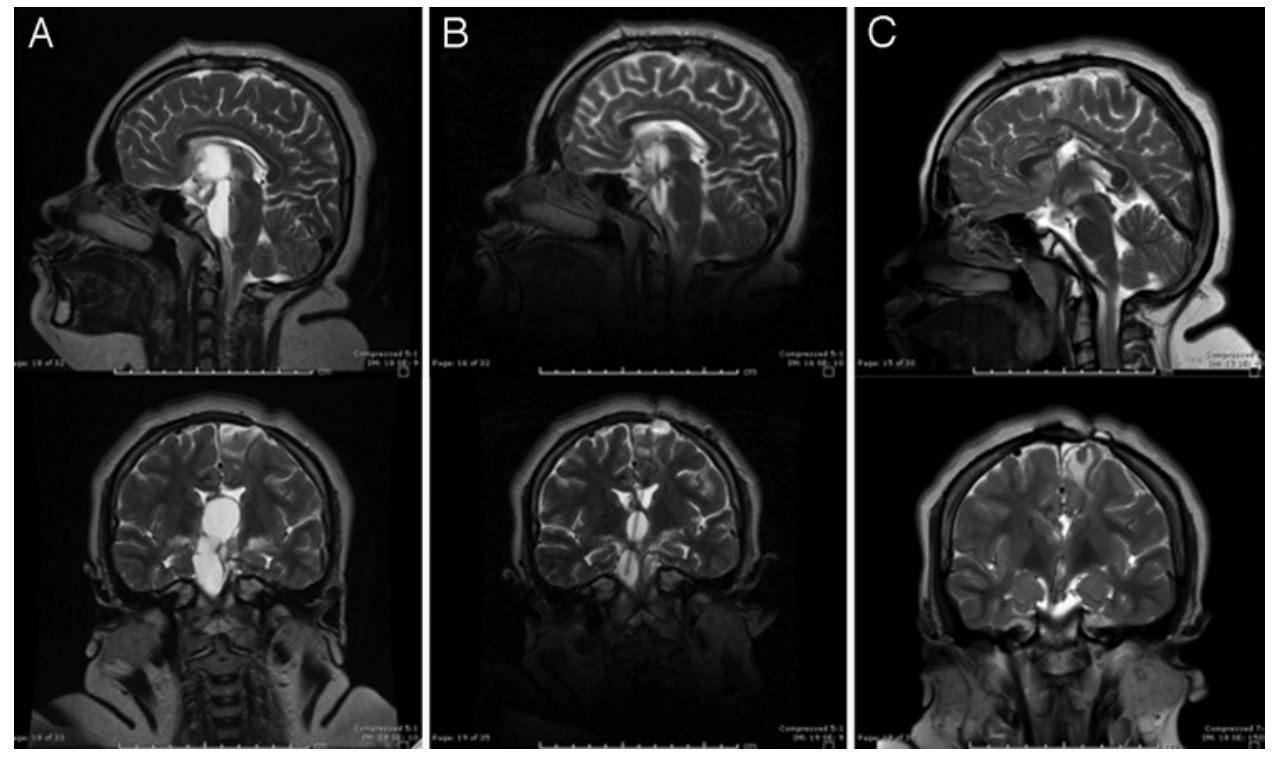

FIG. 6. Stereotactic placement of a catheter into loculated cysts as shown on sagittal (upper) and coronal (lower) MR images. A: Post-IMRT, precatheter placement. B: The catheter within the cysts. C: Cyst resolution 1 year later. 


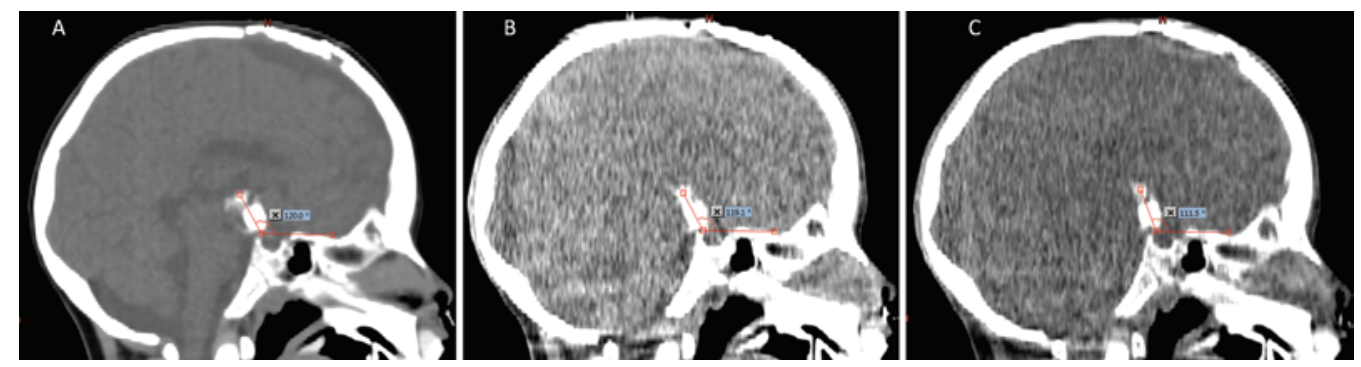

FIG. 7. Daily image guidance with CBCT detects changes in tumor location and size over time. A: Image obtained at CT simulation. B: Image obtained on IMRT treatment Day 1 of 30. C: Image obtained on IMRT treatment Day 20 of 30, which necessitated a subsequent MRI study and plan change.

with pre-radiotherapy MR images provided a method to analyze GTV coverage and reliably adjust treatment plans when significant changes in dosimetry were detected. ${ }^{9}$ When small changes in enhancement, size, or angle are noted in the calcified tumor during daily image review, additional imaging in the form of noncontrast CT, diagnostic MRI, noncontrast MRI, or limited MRI should be performed to detect changes in the target volume and ensure full-dose coverage. In cases in which the GTV coverage falls below $95 \%$ of the intended radiation dose or the PTV falls below $90 \%$ of the total intended dose, adaptive IMRT planning may be required to ensure that the intended IMRT dose covers the entire tumor and cyst volume. If the diagnostic imaging reveals significant change and would result in suboptimal total doses, rapid adaptive planning and/or additional neurosurgical intervention should be considered to minimize breaks from radiation.

At our institution, low-dose CBCT is used for image guidance. Of the 11 patients who demonstrated cyst enlargement at our institution, 4 patients received daily image guidance using CBCT. Each had detectable changes in the calcified components of their tumor by CBCT. While modest in overall imaging quality, CBCT can potentially detect progressive ventriculomegaly and/or tumor calcifications and can provide another tool for the radiation oncologist to detect tumor changes during radiation therapy (Fig. 7).

\section{Conclusions}

In our experience, only Puget Grade 2 tumors had cyst expansion during or in the 12 months following IMRT and had an inferior PFS independent of treatment intent or cyst expansion. Overall, volumetric craniopharyngioma cyst expansion occurred in approximately $40 \%$ of our patients. In the majority of our patients, cyst expansion is a self-limiting process and does not confer a worse outcome. However, sequelae of cyst expansion included progressive obstructive hydrocephalus, compression of the optic chiasm, and clinical decline. When there is solitary cyst expansion, it can be managed with cyst fenestration and aspiration and/or intracystic injectable agents.

In patients with hypothalamic involvement at diagnosis, cyst recurrence or enlargement during IMRT should be carefully monitored with serial diagnostic imaging and can be augmented with image-guided radiation therapy.
In cases in which the PTV falls below $95 \%$ of the total intended dose, adaptive IMRT planning may be required to ensure that the intended IMRT dose covers the entire tumor and cyst volume. Following IMRT, all patients should be closely monitored on routine MR images for solid or cyst progression.

\section{References}

1. Bartels U, Laperriere N, Bouffet E, Drake J: Intracystic therapies for cystic craniopharyngioma in childhood. Front Endocrinol (Lausanne) 3:39, 2012

2. Beltran C, Naik M, Merchant TE: Dosimetric effect of target expansion and setup uncertainty during radiation therapy in pediatric craniopharyngioma. Radiother Oncol 97:399-403, 2010

3. Beltran C, Roca M, Merchant TE: On the benefits and risks of proton therapy in pediatric craniopharyngioma. Int J Radiat Oncol Biol Phys 82:e281-e287, 2012

4. Bishop AJ, Greenfield B, Mahajan A, Paulino AC, Okcu MF, Allen PK, et al: Proton beam therapy versus conformal photon radiation therapy for childhood craniopharyngioma: multi-institutional analysis of outcomes, cyst dynamics, and toxicity. Int J Radiat Oncol Biol Phys 90:354-361, 2014

5. Constine LS, Randall SH, Rubin P, McDonald J: Craniopharyngiomas: fluctuation in cyst size following surgery and radiation therapy. Neurosurgery 24:53-59, 1989

6. Kalapurakal JA: Radiation therapy in the management of pediatric craniopharyngiomas - a review. Childs Nerv Syst 21:808-816, 2005

7. Kiehna EN, Merchant TE: Radiation therapy for pediatric craniopharyngioma. Neurosurg Focus 28(4):E10, 2010

8. Klimo P Jr, Venable GT, Boop FA, Merchant TE: Recurrent craniopharyngioma after conformal radiation in children and the burden of treatment. J Neurosurg Pediatr 15:499-505, 2015

9. Kornguth D, Mahajan A, Frija E, Chang E, Pelloski C, Woo $S$ : Shape variability of craniopharyngioma as measured on CT-on-rails during radiotherapy treatment. Int J Radiat Oncol Biol Phys 66 Suppl:S259-S260, 2006 (Abstract 2091)

10. Merchant TE, Kiehna EN, Kun LE, Mulhern RK, Li C, Xiong X, et al: Phase II trial of conformal radiation therapy for pediatric patients with craniopharyngioma and correlation of surgical factors and radiation dosimetry with change in cognitive function. J Neurosurg 104 (2 Suppl):94-102, 2006

11. Petito CK, DeGirolami U, Earle KM: Craniopharyngiomas: a clinical and pathological review. Cancer 37:1944-1952, 1976

12. Pettorini BL, Inzitari R, Massimi L, Tamburrini G, Caldarelli M, Fanali C, et al: The role of inflammation in the genesis of the cystic component of craniopharyngiomas. Childs Nerv Syst 26:1779-1784, 2010 
13. Puget S, Garnett M, Wray A, Grill J, Habrand JL, Bodaert N, et al: Pediatric craniopharyngiomas: classification and treatment according to the degree of hypothalamic involvement. $\mathbf{J}$ Neurosurg 106 (1 Suppl):3-12, 2007

14. Shi Z, Esiashvili N, Janss AJ, Mazewski CM, MacDonald TJ, Wrubel DM, et al: Transient enlargement of craniopharyngioma after radiation therapy: pattern of magnetic resonance imaging response following radiation. J Neurooncol 109:349-355, 2012

15. Stripp DCH, Maity A, Janss AJ, Belasco JB, Tochner ZA, Goldwein JW, et al: Surgery with or without radiation therapy in the management of craniopharyngiomas in children and young adults. Int J Radiat Oncol Biol Phys 58:714-720, 2004

16. Winkfield KM, Linsenmeier C, Yock TI, Grant PE, Yeap BY, Butler WE, et al: Surveillance of craniopharyngioma cyst growth in children treated with proton radiotherapy. Int J Radiat Oncol Biol Phys 73:716-721, 2009

17. Winkfield KM, Tsai HK, Yao X, Larson E, Neuberg D, Pomeroy SL, et al: Long-term clinical outcomes following treatment of childhood craniopharyngioma. Pediatr Blood Cancer 56:1120-1126, 2011

\section{Disclosures}

The authors report no conflict of interest concerning the materials or methods used in this study or the findings specified in this paper.

\section{Author Contributions}

Conception and design: Kiehna, Wong. Acquisition of data: Lamiman. Analysis and interpretation of data: Kiehna, Lamiman,

Wong, Tamrazi. Drafting the article: Lamiman. Critically revising the article: all authors. Reviewed submitted version of manuscript: Kiehna, Lamiman, Wong. Approved the final version of the manuscript on behalf of all authors: Kiehna. Statistical analysis: Lamiman. Study supervision: Kiehna.

\section{Correspondence}

Erin Kiehna, Department of Neurosurgery, Children's Hospital Los Angeles, Keck School of Medicine of USC, 1300 N Vermont Ave., Ste. 1006, Los Angeles, CA 90027. email: ekiehna@chla.usc.edu. 